\title{
Ci può essere qualcosa di inarrivabile
}

\author{
Paolo D'Achille
}

PUBBLICATO: 11 SETTEMBRE 2020

\section{Quesito:}

Ci sono alcuni che ritengono che l'aggettivo inarrivabile sia scorretto, sia perché il suffisso -bile si lega a verbi transitivi, sia perché manca la forma positiva arrivabile, e ritengono che al suo posto andrebbe usato irraggiungibile.

\section{Ci può essere qualcosa di inarrivabile}

$\mathrm{L}^{\prime}$

9 aggettivo inarrivabile 'difficile da raggiungere' e, forse ancora più spesso, 'difficile da eguagliare' è registrato in tutti i principali dizionari italiani ed è datato i632 sia nel GRADIT, sia nello Zingarelli 202I, sulla base del primo esempio riportato nel GDLI s.v. inarrivabile, in cui il significato dell'aggettivo è quello, oggi raro, di 'difficile da comprendere' ("Vero è che qui non si trattano cose da me desideratissime, [...] e molte altre cose ch'io stimo inarrivabili mentre Vostra Signoria le tace", da una lettera di Tommaso Campanella a Galileo Galilei). All'interno del GDLI l'aggettivo ha oltre 70 occorrenze, che documentano una indubbia continuità d'uso nel corso dei secoli. Sulla base di Google libri è possibile perfino anticiparne il "certificato di nascita", visto che inarrivabile è attestato nel Mazzetto di varj discorsi sacri dell'eremitano Giovanni Paolo Caprini, edito postumo a Napoli nel I6r3, che parla "della inarrivabile patienza Divina" (pp. 382-383). Già questi dati basterebbero a legittimarne l'uso.

Ma anche le due obiezioni avanzate dai lettori, a un esame attento, cadono: l'aggettivo arrivabile, nel senso di 'raggiungibile' e 'comprensibile', è infatti documentato anch'esso, sebbene molto meno spesso (il GRADIT lo marca come di B[asso]U[so], mentre dà a inarrivabile l'etichetta CO[mune]) e solo posteriormente: la data riportata nello stesso dizionario è av. I704 e si basa sull'unico esempio registrato, s.v. arrivabile, dal GDLI, che a sua volta marca l'aggettivo come Disus[ato] ("In questo mondo si dipende dalla necessità, che nulla intende, e par che tutto governi con intelligenza non arrivabile", Lorenzo Bellini; si noti la presenza della negazione). Ma la data offerta dallo Zingarelli 202I è r624 (posteriore comunque, seppur di poco, al r6rz di inarrivabile).

Ora, la circostanza che gli aggettivi positivi in -bile siano spesso attestati più tardi e comunque usati meno frequentemente di quelli negativi, che pure si considerano derivati dai precedenti grazie al prefisso in-, è stata già rilevata e spiegata, nella sua esemplare trattazione del suffisso, da Davide Ricca (in Grossmann-Rainer 2004, pp. 422-429). Secondo Ricca, "il derivato negativo denota spesso una proprietà molto più stabile della corrispondente positiva, quindi più adatta ad essere lessicalizzata con un aggettivo (cfr. [...] imperturbabile, introvabile), oppure una proprietà comunicativamente molto più informativa (come per imperdibile, imprendibile o inaffondabile). Le rispettive formazioni positive, quindi, andranno meglio considerate come marginali nell'uso anziché impossibili" (p. 425). Alcune di esse, inoltre, possono essere considerate delle retroformazioni (cioè formazioni che hanno applicato al contrario il processo di derivazione: sulla base dell'aggettivo negativo si è formato quello positivo, senza il prefisso in-). La stessa cosa, aggiungiamo, è avvenuta con aggettivi in -bile che sono in realtà latinismi: si pensi a immarcescibile 'che non puo marcire', 'imperituro', calcato sul latino immarcescibilis, da cui si è ricavato marcescibile 'che può marcire', 'deperibile', documentato più tardi ( 5584 e r625 sono 
le datazioni fornite nello Zingarelli 202I, che peraltro ritiene che il processo di retroformazione sia avvenuto in latino, visto che esiste anche marcescibilis, considerato alla base di marcescibile).

Quanto al fatto che -bile si aggiunga in questo caso a un verbo intransitivo come arrivare, lo stesso Ricca non solo ha precisato che non tutti i verbi transitivi ammettono aggettivi in -bile, ma ha segnalato anche la possibilità, sia pure eccezionale, di formazioni tratte da verbi intransitivi, come deperibile, fallibile, stabile; questa derivazione è un po' più frequente proprio per gli aggettivi prefissati i n in- come "immancabile, imprescindibile, inarrivabile, incrollabile, inservibile, intramontabile, i cui corrispondenti positivi paiono davvero poco accettabili, accanto a casi come irresistibile, irrinunciabile, dove i positivi esistono ma sono con buona probabilità retroformazioni più recenti" (p. 426). Data l'esistenza di arrivabile, inarrivabile andrebbe spostato in questo secondo gruppo.

D'altra parte, che il suffisso -bile abbia valicato i propri ambiti d'uso originari è dimostrato da formazioni in cui si aggiunge non a verbi ma a nomi, come camionabile (strada camionabile o camionale, che può essere percorsa da camion) e carrabile (passo carrabile o carraio, tratto di strada o di marciapiede che va lasciato libero perché adibito al passaggio di veicoli ), registrate nello Zingarelli 202r con le date igr9 e I942.

Naturalmente, i nostri lettori possono senz'altro continuare a sostituire inarrivabile, a loro poco gradito, con irraggiungibile ( $\mathrm{ma}$ in certi casi l'equivalente sarà invece impareggiabile o, talvolta, incomprensibile), ma che l'aggettivo sia corretto è fuor di dubbio.

\section{Cita come:}

Paolo D'Achille, Ci può essere qualcosa di inarrivabile, "Italiano digitale", 2020, XIV, 2020/3 (lugliosettembre)

DOI: $10.35948 / 2532-9006 / 2020.4391$

\section{Copyright 2020 Accademia della Crusca}

Pubblicato con licenza creative commons CC BY-NC-ND 